\title{
Cutibacterium modestum and "Propionibacterium humerusii" represent the same species that is commonly misidentified as Cutibacterium acnes
}

\author{
Daniel Goldenberger • Kirstine K. Søgaard • Aline Cuénod · Helena Seth-Smith • \\ Daniel de Menezes $\cdot$ Peter Vandamme $\cdot$ Adrian Egli
}

Received: 4 March 2021 / Accepted: 26 April 2021 / Published online: 7 May 2021

(C) The Author(s) 2021

\begin{abstract}
Cutibacterium spp. play an increasing role in soft tissue and implant-associated infections. We isolated a novel Cutibacterium spp. from an implant and investigated this isolate using multiple identification approaches. Correct identification was hampered by inconsistent reference data. The isolate was characterised using conventional methods such as Gram stain, MALDI-TOF MS, and antimicrobial susceptibility testing against multiple antimicrobials. Partial $16 \mathrm{~S}$ rRNA gene sequencing and whole genome
\end{abstract}

Supplementary Information The online version contains supplementary material available at https://doi.org/10.1007/ s10482-021-01589-5.

D. Goldenberger $(\bowtie) \cdot$ K. K. Søgaard .

H. Seth-Smith · A. Egli

Division of Clinical Bacteriology and Mycology,

University Hospital Basel, University of Basel,

Petersgraben 4, 4031 Basel, Switzerland

e-mail: daniel.goldenberger@usb.ch

K. K. Søgaard · A. Cuénod · H. Seth-Smith · A. Egli

Applied Microbiology Research, Department of

Biomedicine, University of Basel, Basel, Switzerland

D. de Menezes

Clinic of Orthopedy, Spitalzentrum Biel, Biel-Bienne,

Switzerland

P. Vandamme

Laboratory of Microbiology, Department of Biochemistry and Biotechnology, Faculty of Sciences, Ghent

University, Ghent, Belgium sequencing were also performed. In addition, we summarised the available published sequence data and compared prior data to our strain. Conventional phenotypic identification of our isolate resulted in Cutibacterium spp. After analysis of 16S rRNA gene and genome sequences, our isolate was identified as $C$. modestum, a very recently described species. The $16 \mathrm{~S}$ rRNA gene analysis was hampered by three incorrect nucleotides within the 16S rRNA gene reference sequence of $C$. modestum $\mathrm{M}^{\mathrm{T}}$ (accession no. LC466959). We also clearly demonstrate that this novel species is identical to tentatively named "Propionibacterium humerusii”. Retrospective data

\footnotetext{
P. Vandamme

BCCM/LMG Bacteria Collection, Department of

Biochemistry and Biotechnology, Faculty of Sciences,

Ghent University, Ghent, Belgium
} 
analysis indicates that $C$. modestum is a clinically important Cutibacterium species often misidentified as $C$. acnes. The isolation and identification of Cutibacterium spp. is still a challenge. The correct description of very recently named $C$. modestum and the availability of a correct 16S rRNA sequence of the type strain may help to clarify the taxonomical uncertainty concerning " $P$. humerusii". The novel C. modestum is an additional, clinically important species within the genus Cutibacterium and may represent a new member of the human skin microbiome.

Keywords Clinical significance $\cdot$ Cutibacterium acnes - Cutibacterium modestum - Genome analysis . "Propionibacterium humerusii" · Taxonomy

\section{Introduction}

In 2016, the genus Propionibacterium was restructured on the basis of genomic evidence, and separated into Propionibacterium sensu stricto and three novel genera: Acidipropionibacterium, Cutibacterium, and Pseudopropionibacterium (Scholz and Kilian 2016). The cutaneous species earlier classified as Propionibacterium acnes, $P$. avidum, $P$. granulosum, and $P$. namnetense (Aubin et al. 2016) were assigned to the new genus Cutibacterium. While $C$. acnes is wellknown for its potential to cause acne vulgaris, postsurgical infections and other human infections, less is known for the other species. In 2011, a novel Propionibacterium species was reported in a patient with humeral infection after revision of a total shoulder arthroplasty. This new species was tentatively named " $P$. humerusii" based on genomic data (Butler-Wu et al. 2011). Very recently, a Japanese group described an isolate from a patient with inflamed meibomian glands for which they formally proposed the name Cutibacterium modestum sp. nov. (Dekio et al. 2020).

Here, we describe a novel clinical isolate belonging to Cutibacterium modestum from a patient with an infected hip implant. During our analyses of this strain and retrospective data analysis of similar 16S rRNA gene and whole genome sequences, we found evidence that " $P$. humerusii" and $C$. modestum represent the same species and that this species is often misidentified as $C$. acnes.

\section{Material and methods}

Culture, identification methods, and antimicrobial susceptibility testing (AST)

Aerobic and anaerobic culture was performed according routine microbiological procedures. For tentative identification, we compared the obtained spectra from the MALDI-TOF MS (microflex LT, Bruker Daltonics) to the current MALDI-TOF database version (MBT 8468 MSP Library, BDAL V9.0.0.0_78548468). Partial 16S rRNA gene sequencing was executed as described previously (Hinic et al. 2014) and AST was performed using the gradient diffusion technology (MIC Test Strip; Liofilchem) against 20 antimicrobial agents under anaerobic conditions.

Genome sequencing, assembly and phylogenetic analysis

DNA extracted from isolate 602588-20-USB was sequenced on the Illumina NextSeq platform (PE150) following library creation with Nexteraflex (Illumina). Digital DNA-DNA hybridisation (dDDH) used GGDC2.1 (http://ggdc.dsmz.de/ggdc.php\#) and the DDH cut off of $<70 \%$ ) (Auch et al. 2010). Reads (mean $92 \times$ coverage) were assembled using Unicycler (Wick et al. 2017) to produce an assembly of 2.6 $\mathrm{Mb}$ in 22 contigs. The neighbour joining tree using whole genome SNPs was created in CLC genomic workbench v20.0.2 using parameters described in the figure legend (Fig. 1).

Retrospective sequence analysis

Very similar 16S rRNA gene sequences and genome sequences associated with $C$. modestum were retrieved using the BLAST algorithm and were compared with the sequences from $C$. modestum strain $\mathrm{M} 12^{\mathrm{T}}$ and our isolate 602588-20-USB. 


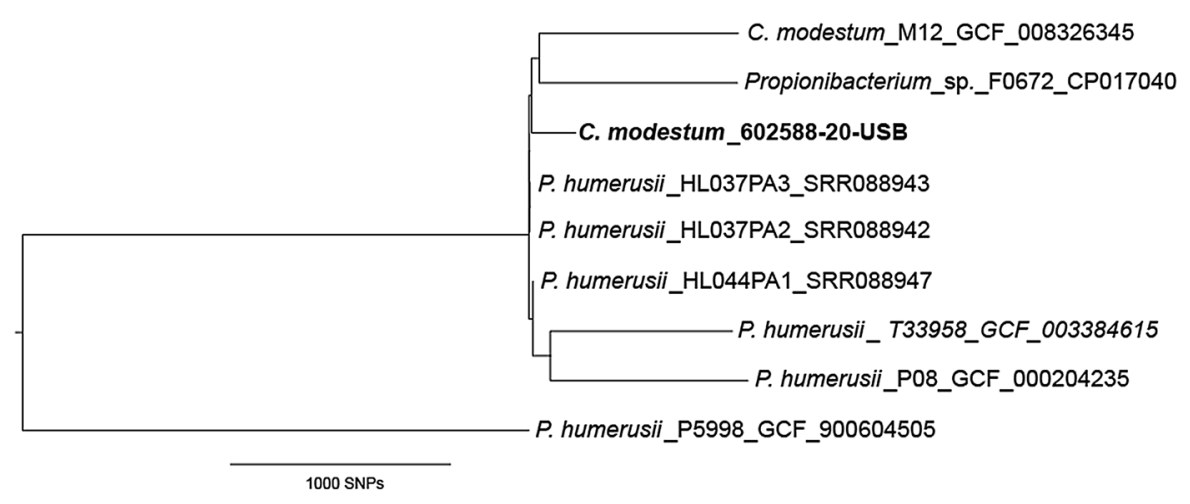

Fig. 1 Whole genome SNP tree comparing isolates belonging to " $P$. humerusii" and C. modestum Neighbour joining SNP tree created using CLC genomic workbench v20.0.2. All relevant genomes were downloaded from NCBI, accession numbers are given in the figure. The newly described isolate genome is in bold. Tree rooted using the genome of the type strain of $C$. acnes: ATCC6919 (GCA_003030305, not shown). Mapping, variant calling and single nucleotide polymorphism (SNP) tree creation used parameters that differed from the default as:

\section{Results}

A 39 year old male patient presented with an implantrelated hip infection following internal fixation of a femoral neck fracture. After debridement surgery and antibiotic treatment of Staphylococcus capitis according to susceptibility testing, no signs of persistent infection were identified at the 6 months postoperative control. C. modestum was not covered by antimicrobial therapy. From three cannulated screws sent for culture, growth of Staphylococcus capitis $(>1000$ $\mathrm{CFU} / \mathrm{ml})$ and Cutibacterium species $(80 \mathrm{CFU} / \mathrm{ml})$ was observed. After six days of anaerobic incubation on BD Brucella blood agar (Becton Dickinson), we detected characteristic white colonies and corresponding typical Gram-positive rods that was compatible with presumptive identification of $C$. acnes. Catalase and indole reactions were positive and confirmed this suggestion. MALDI-TOF was not able to provide valid identification. The first three MALDI-TOF hits were Propionibacterium sp. score 1.64, C. acnes score 1.61 and $C$. acnes 1.51. Subsequent partial 16S rRNA gene analysis (693 bp) showed 100\% identity to 9 sequences of Propionibacterium acnes / Propionibacterium spp./ "P. humerusii" isolates, followed by identities to type reference sequences of $C$. acnes ATCC6919 (97.7\%), C. namnetense NTS31307302 (97.3\%), C. avidum ATCC25577 (95.1\%) and C. granulosum DSM20700 (93.4\%). AST showed that variant calling with single ploidy, $10 \times$ minimum coverage, 10 minimum count and $70 \%$ minimum frequency, and SNP tree creation with $10 \times$ minimum coverage, $10 \%$ minimum coverage, 0 prune distance and including multi-nucleotide variants (MNVs). Where required, assemblies were shredded into reads using SAMtools wgsim prior to phylogeny construction. The genome of T33958 was used as the reference (italics) as the M12 assembly is in nine contigs. All data mapped to over $97 \%$ of the reference assembly

isolate 602588-20-USB had similar patterns to other Cutibacterium spp., thus susceptible to common antimicrobial substances, whereas it was resistant to metronidazole and gentamicin (see supplementary materials table $\mathrm{S} 1$ ).

Retrospective analysis of $16 \mathrm{~S}$ rRNA gene sequences in public databases resulted in 11 entries with highly similar (99.2-100\%) 16S rRNA gene

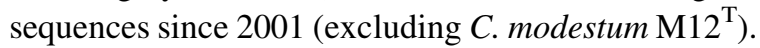
The corresponding isolates originated from different countries and sources showing that this organism has a clinical relevance (Table 1), (Dekio et al. 2020; Kunishima et al. 2001; Lin et al. 2010). Five of these 11 isolates were identified as $C$. acnes despite considerable differences (97.5-98.2\%) towards the 16S rRNA gene sequence of the $C$. acnes type strain (ATCC $6919^{\mathrm{T}}$ ). We also detected within the 16S rRNA gene sequence of $C$. modestum $\mathrm{M} 12^{\mathrm{T}}$ (LC466959) three mismatches compared to the whole-genome-sequence of M12 ${ }^{\mathrm{T}}$ (BJEN01000000): position 3 a $\mathrm{G}$ instead of $\mathrm{A}$, position 11 a $\mathrm{T}$ instead of $\mathrm{C}$, and position 1484 an A instead of G (Dekio et al. 2020) (see also Table 1).

Genome sequences of six additional " $P$. humerusii" strains are present in public databases (Table 2), (Butler-Wu et al. 2011; Dekio et al. 2020). Table 2 also includes genome sequence data of our isolate 602588-20-USB, C. modestum strain $\mathrm{M} 12^{\mathrm{T}}$, and $C$. acnes ATCC $6919^{\mathrm{T}}$ for comparison. 16S rRNA gene 
Table 1 List of probable C. modestum isolates $(\mathrm{n}=13)$

\begin{tabular}{|c|c|c|c|c|c|c|c|}
\hline \multirow[t]{2}{*}{ Strain } & \multirow[t]{2}{*}{ Specimen } & \multirow[t]{2}{*}{ Country } & \multirow[t]{2}{*}{ Year } & \multirow[t]{2}{*}{ Accession no } & \multicolumn{2}{|c|}{ 16S rRNA gene } & \multirow[t]{2}{*}{ References } \\
\hline & & & & & (bp) & $(\%)$ & \\
\hline \multicolumn{8}{|c|}{ Incorrectly labeled as Propionibacterium acnes, $n=5$} \\
\hline 7375 & Blood component & Japan & 2001 & AB042290 & $1482 / 1483$ & 99.9 & Kunishima et al. (2001) \\
\hline 8800 & Blood component & Japan & 2003 & AB108481 & $1480 / 1483$ & 99.8 & unpubl \\
\hline L340 & "Clinical strain “ & Taiwan & 2010 & GQ496494 & $1410 / 1421^{\mathrm{a}}$ & 99.2 & Lin et al. (2010) \\
\hline NN1204 & Pus & China & 2015 & KP944185 & $1418 / 1421$ & 99.8 & unpubl \\
\hline JK19.3 & Cardiac pacemaker & Japan & 2017 & LC341281 & $1349 / 1349$ & 100 & unpubl \\
\hline \multicolumn{8}{|c|}{ "P. humerusii", $n=1$} \\
\hline R7A_C5_IA & Surface from ISS & Austria & 2019 & LR215132 & $1275 / 1277$ & 99.8 & unpubl \\
\hline \multicolumn{8}{|c|}{ Propionibacterium/Cutibacterium sp., $n=7$} \\
\hline Met-C3 & Dental plaque & USA & 2009 & GQ422729 & $1484 / 1485$ & 99.9 & unpubl \\
\hline P5998 & Vagina & France & 2018 & LT996136 & $1486 / 1486$ & 100 & unpubl \\
\hline F0672 & Oral microbiome & USA & 2018 & СР017040 & $1486 / 1486$ & 100 & unpubl \\
\hline KB17-24,694 & Blood & Japan & 2018 & LC414574 & $1366 / 1366$ & 100 & unpubl \\
\hline $\mathrm{M} 12^{\mathrm{T}}$ & Meibomian glands & Japan & 2019 & LC466959 & $1483 / 1486^{\mathrm{b}}$ & 99.8 & Dekio et al. (2020) \\
\hline NM47_B9-13 & Murine gut & Canada & 2019 & MK929068 & $1486 / 1486$ & 100 & unpubl \\
\hline $\begin{array}{l}\text { 602588-20- } \\
\text { USB }\end{array}$ & Implant hip & Switzerland & 2020 & HG992826 & $1486 / 1486$ & 100 & Present study \\
\hline
\end{tabular}

Database analysis based on BLAST analysis with parameters: "nucleotide collection, exclude uncultured"; based on extracted $C$. modestum 16S rRNA gene sequence of $\mathrm{M} 12^{\mathrm{T}}$, accession no. BJEN0100000

${ }^{a}$ Main nucleotide differences at the end of the sequence indicating probable sequencing errors

${ }^{\mathrm{b}}$ Strain $\mathrm{M} 12^{\mathrm{T}}$ : $16 \mathrm{~S}$ rRNA sequence shows 3 nucleotide differences compared with extracted 16S rRNA sequence of the genome

sequences extracted from the " $P$. humerusii", 602588-20-USB and C. modestum $\mathrm{M} 12^{\mathrm{T}}$ genomes were $100 \%$ identical and $\mathrm{dDDH}$ and ANI values indicated that these organisms represented a single species, i.e. C. modestum. In Fig. 1, a whole genome SNP phylogenetic tree of the isolates listed in Table 2 is presented and reveales a high genomic homogeneity among all $C$. modestum isolates.

In addition, we retrieved more than 100 sequence database entries of uncultured bacterium clone sequences from the human skin with 99.9-100\% identity of a length of $1330 \mathrm{bp}$ or longer compared to extracted $C$. modestum $16 \mathrm{~S}$ rRNA sequence $\mathrm{M} 12^{\mathrm{T}}$. These sequences all belong to human skin microbiome data from three different studies (Grice et al. 2009; Kong et al. 2012; Oh et al. 2013).

\section{Genome data availability}

Genome data is deposited in ENA project PRJEB41775 and the 16SrRNA gene sequence under accession no. HG992826.

\section{Discussion}

We report a novel clinical isolate belonging to the recently described C. modestum (Dekio et al. 2020). Correct species identification was enabled only by partial 16S rRNA gene sequencing and whole genome analysis. Unfortunately, the 16S rRNA gene analysis was hampered by three incorrect nucleotides within the 16S rRNA reference sequence of $C$. modestum M12 ${ }^{\mathrm{T}}$ (accession no. LC466959), and incorrect $C$. acnes species designations in multiple 16S rRNA sequence entries.

The catalase test is an important biochemical characteristic for preliminary identification of Cutibacterium sp. Despite performing API Coryne 


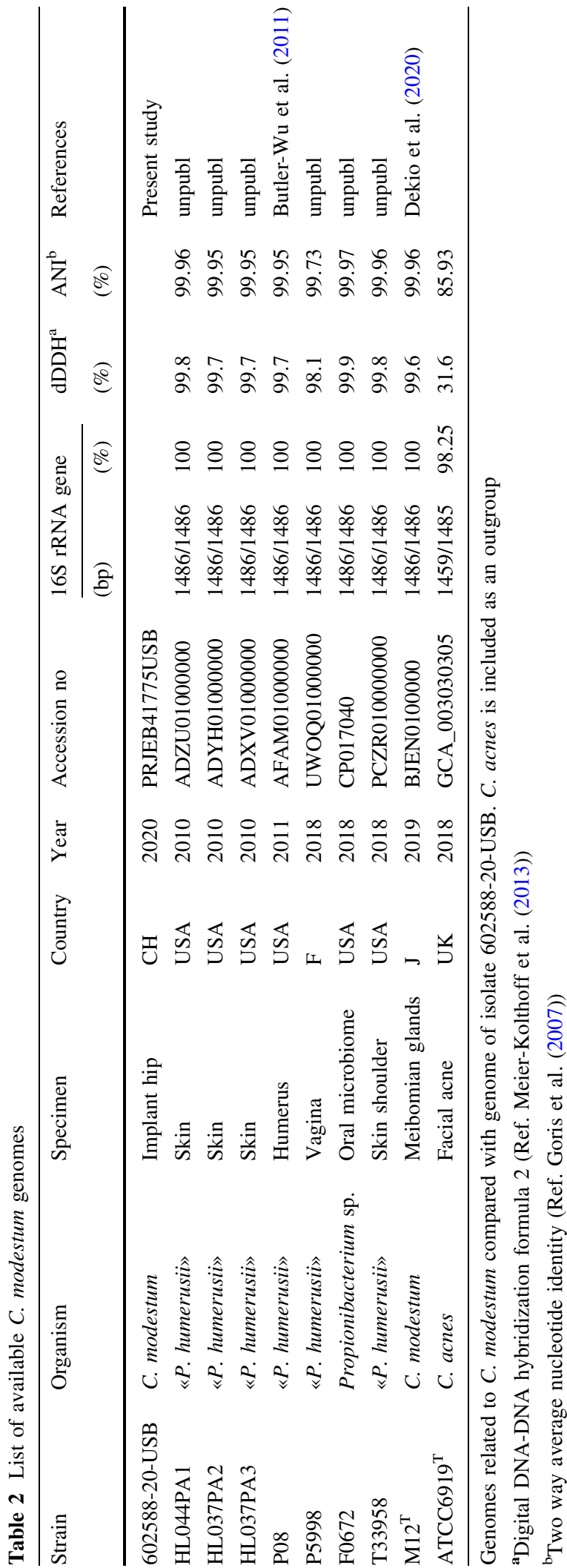

analysis that comprises the catalase reaction, this result was not reported in the taxonomic proposal of $C$. modestum as a novel species (Dekio et al. 2020). The indole test is another basic biochemical reaction for biochemical identification of Cutibacterium sp. which was reported negative in the Dekio et al. study (Dekio et al. 2020). In contrast, our strain showed a clear positive indole and positive catalase reaction which confirms data reported for the tentatively characterised "P. humerusii" strain (Butler-Wu et al. 2011).

Routine MALDI-TOF MS failed to identify $C$. modestum because this species is not yet recorded in the commercial database. Dekio et al. (2020) reported four predominant MALDI-TOF MS peaks at 3493, 3712, 6986 and $7424 \mathrm{Da}$ in C. modestum $\mathrm{M} 12^{\mathrm{T}}$. The same $m / z$ peaks were also present in the spectrum of our isolate confirming their diagnostic value for future identification of $C$. modestum with MALDI-TOF MS (see supplementary materials figure S2).

The retrospective analysis of $16 \mathrm{~S}$ rRNA sequence entries showed that $C$. modestum represents a bacterial organism of considerable clinical significance which often has been misidentified as $C$. acnes. Unfortunately, these incorrect data may lead to further misidentifications in current diagnostic applications based on 16S rRNA gene BLAST analysis.

Comparative analysis of the available genomes of this bacterium clearly indicates that $C$. modestum and " $P$. humerusii" represent the same species and consequently the " $P$. humerusii" database entries should be renamed (Table 2). Surprisingly, the corresponding phylogenetic tree only shows minimal genomic differences with exception of strain P5998 among the $C$. modestum and "P. humerusii" strains despite the origin of the isolates from different continents (Fig. 1).

We demonstrate for the first time that novel $C$. modestum might represent an organism of the normal skin microbiota. The high similarity to multiple uncultured clone sequences could indicate that this organism is difficult to detect using conventional cultural methods.

To conclude, the isolation and identification of Cutibacterium spp. remains challenging. The correct description of very recently named $C$. modestum and the availability of a correct $16 \mathrm{~S}$ rRNA sequence of the type strain may help to clarify the taxonomical uncertainty concerning " $P$. humerusii". C. modestum is identical to the previously named " $P$. humerusii" 
and represents a further clinically important species within the genus Cutibacterium.

Acknowledgements We thank Christine Kiessling for excellent NGS technical support. Genome assemblies were performed at sciCORE (http://scicore.unibas.ch/) scientific computing center at University of Basel.

Author contributions DG, PV: Methodology and data validation. AC: MALDI TOF MS analysis. HSS: Genome analysis, writing original draft. DdM: Providing clinical sample and clinical data. DG: Conceptualisation, supervision, writing original draft. KKS: Methodology, writing original draft. AE: Supervision. All authors reviewed the original draft.

Funding Open Access funding provided by Universität Basel (Universitätsbibliothek Basel). This work received no public or private funds.

Availability of data and material Sequences obtained in this study have been deposited in ENA Project PRJEB41775 and the $16 \mathrm{~S}$ rRNA gene sequence is found under assession no. HG992826. The datasets used and/or analysed during the current study are available from the corresponding author on reasonable request.

\section{Declarations}

Conflict of interest The authors declare that the research was conducted in the absence of any commercial or financial relationships that could be construed as a potential conflict of interest.

Consent to participate The patient's consent was obtained.

Open Access This article is licensed under a Creative Commons Attribution 4.0 International License, which permits use, sharing, adaptation, distribution and reproduction in any medium or format, as long as you give appropriate credit to the original author(s) and the source, provide a link to the Creative Commons licence, and indicate if changes were made. The images or other third party material in this article are included in the article's Creative Commons licence, unless indicated otherwise in a credit line to the material. If material is not included in the article's Creative Commons licence and your intended use is not permitted by statutory regulation or exceeds the permitted use, you will need to obtain permission directly from the copyright holder. To view a copy of this licence, visit http://creativecommons.org/licenses/by/4.0/.

\section{References}

Aubin GG, Bemer P, Kambarev S, Patel NB, Lemenand O, Caillon J et al (2016) Propionibacterium namnetense sp. nov., isolated from a human bone infection. Int J Syst Evol Microbiol 66(9):3393-9
Auch AF, von Jan M, Klenk H-P, Goker M (2010) Digital DNADNA hybridization for microbial species delineation by means of genome-to-genome sequence comparison. Stand Genom Sci 2(1):117-34

Butler-Wu SM, Sengupta DJ, Kittichotirat W, Matsen FA 3rd, Bumgarner RE (2011) Genome sequence of a novel species, Propionibacterium humerusii. J Bacteriol 193(14):3678

Dekio I, Sakamoto M, Suzuki T, Yuki M, Kinoshita S, Murakami Y et al (2020) Cutibacterium modestum sp. nov., isolated from meibum of human meibomian glands, and emended descriptions of Cutibacterium granulosum and Cutibacterium namnetense. Int J Syst Evol Microbiol 70(4):2457-62

Goris J, Konstantinidis KT, Klappenbach JA, Coenye T, Vandamme P, Tiedje JM (2007) DNA-DNA hybridization values and their relationship to whole-genome sequence similarities. Int J Syst Evol Microbiol 57(Pt 1):81-91

Grice EA, Kong HH, Conlan S, Deming CB, Davis J, Young AC et al (2009) Topographical and temporal diversity of the human skin microbiome. Science 324(5931):1190-2

Hinic V, Aittakorpi A, Suter S, Turan S, Schultheiss E, Frei R et al (2014) Evaluation of the novel microarray-based prove-it bone \& joint assay for direct detection of pathogens from normally sterile body sites in comparison with culture and broad-range bacterial PCR. J Microbiol Methods 107:38-40

Kong HH, Oh J, Deming C, Conlan S, Grice EA, Beatson MA et al (2012) Temporal shifts in the skin microbiome associated with disease flares and treatment in children with atopic dermatitis. Genome Res 22(5):850-9

Kunishima S, Inoue C, Kamiya T, Ozawa K (2001) Presence of Propionibacterium acnes in blood components. Transfusion 41(9):1126-9

Lin YT, Vaneechoutte M, Huang AH, Teng LJ, Chen HM, Su SL et al (2010) Identification of clinically important anaerobic bacteria by an oligonucleotide array. J Clin Microbiol 48(4):1283-90

Meier-Kolthoff JP, Auch AF, Klenk H-P, Goker M (2013) Genome sequence-based species delimitation with confidence intervals and improved distance functions. BMC Bioinform 14:60

Oh J, Freeman AF, Program NCS, Park M, Sokolic R, Candotti F et al (2013) The altered landscape of the human skin microbiome in patients with primary immunodeficiencies. Genome Res 23(12):2103-14

Scholz CFP, Kilian M (2016) The natural history of cutaneous propionibacteria, and reclassification of selected species within the genus Propionibacterium to the proposed novel genera Acidipropionibacterium gen. nov., Cutibacterium gen. nov. and Pseudopropionibacterium gen. nov. Int $\mathbf{J}$ Syst Evol Microbiol 66(11):4422-32

Wick RR, Judd LM, Gorrie CL, Holt KE (2017) Unicycler: resolving bacterial genome assemblies from short and long sequencing reads. PLoS Comput Biol 13(6):e1005595

Publisher's Note Springer Nature remains neutral with regard to jurisdictional claims in published maps and institutional affiliations. 